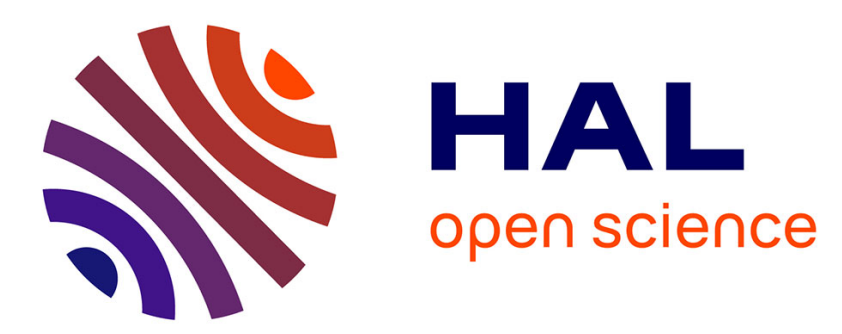

\title{
Comparison of different gaits with rotation of the feet for a planar biped
}

\author{
David Tlalolini, Christine Chevallereau, Yannick Aoustin
}

\section{To cite this version:}

David Tlalolini, Christine Chevallereau, Yannick Aoustin. Comparison of different gaits with rotation of the feet for a planar biped. Robotics and Autonomous Systems, 2009, 57 (4), pp.371-383. 10.1016/j.robot.2008.09.008 . hal-00794718

\section{HAL Id: hal-00794718 https://hal.science/hal-00794718}

Submitted on 26 Feb 2013

HAL is a multi-disciplinary open access archive for the deposit and dissemination of scientific research documents, whether they are published or not. The documents may come from teaching and research institutions in France or abroad, or from public or private research centers.
L'archive ouverte pluridisciplinaire HAL, est destinée au dépôt et à la diffusion de documents scientifiques de niveau recherche, publiés ou non, émanant des établissements d'enseignement et de recherche français ou étrangers, des laboratoires publics ou privés. 


\title{
Comparison of different gaits with rotation of the feet for a planar biped
}

\author{
D. Tlalolini, C. Chevallereau, Y. Aoustin \\ IRCCyN, UMR 6597, CNRS, Ecole Centrale de Nantes, Université de Nantes \\ BP 92101, 1 rue de la Noë, 44321 Nantes cedex 03
}

\begin{abstract}
Fast human walking includes a phase where the stance heel rises from the ground and the stance foot rotates about the stance toe. This phase where the biped becomes under-actuated is not present during the walk of humanoid robots. The objective of this study is to determine if this phase is useful to reduce the energy consumed in the walking. In order to study the efficiency of this phase, six cyclic gaits are presented for a planar biped robot. The simplest cyclic motion is composed of successive single support phases with flat stance foot on the ground. The most complex cyclic motion is composed of single support phases that include a subphase of rotation of the stance foot about the toe and of finite time double support phase. For the synthesis of these walking gaits, optimal motions with respect to the torque cost, are defined by taking into account given performances of actuators. It is shown that for fast motions a foot rotation sub-phase is useful to reduce the criteria cost. In the optimization process, under-actuated phase (foot rotation phase), fullyactuated phase (flat foot phase) and over-actuated phase (double support phase) are considered.
\end{abstract}

Key words:

bipedal robot, optimal motion, under-actuated system, over-actuated system, walking gait.

\section{Introduction}

The interests to design reference trajectories for dynamical stable walking bipeds are to improve the fluidity of the desired motion and to help in the conception of an experimental device. In general, this open and non-trivial problem is solved by finding numerical solutions. Optimization techniques are very often used to obtain low-energy trajectories for biped robots. 
One of the first papers in this topic is (9) where Chow and Jacobson shown that the optimization methods are efficient to study the human locomotion. In (2), Beletski et al used polynomial functions of time to optimize the joint trajectories of a fully-actuated planar biped in single support. The optimization variables are the coefficients of polynomial functions and a simplified dynamical model is used. Later in (5), (4) authors used the same techniques of parametric optimization for more sophisticate bipeds to find coefficients respectively for polynomial functions and finite Fourier series and to obtain a low-energy cyclic gait. In (18), (14) the torques are optimized variables. Then the joint coordinates are found by solving the direct dynamic problem and by integration. Equality constraints are added in order to insure cyclic gaits. Other techniques are also available to define reference trajectory. In (16) blending or interpolation have been used for planar motion. With this strategy, (13) shows that the Balman model suffices to do a somersault motion for models with low degrees of freedom, using only four discrete points in time. However with these methods no energy criteria can be taken into account.

In the design of reference trajectories, the biped robots can present underactuated phases (7), (24), (10), (23), over-actuated phases (17), (15) or completely actuated phase (12).

From studies of human walking gaits, authors proved the fundamental role of the feet during the walking gait in double support phases and in single support phases. Thus, for human walking gaits in single support, a rotation of the foot is observed with a partial contact of the sole with the ground, located between the heel and the toe (20). Furthermore it is shown that the feet, with joint torques at the ankle which are significant, play a role more important to insure an equilibrium of the biped than to help the locomotion (22), (21), (1). Thus it is extremely important and interesting to study the walking gait of an anthropomorphic biped with rotation of the feet. For a seven-link biped, an optimized walking gait composed of single support phases and double support phases is proposed in (11), (3). In single support phase the biped is flat on the ground, the rotation of the feet appears in double support phase only. An impactless heel-touch of the swing leg is taken into account at the beginning of the double support phase.

In this paper, we propose to take into account of a rotation foot during the single support phase. The objective of this study is to determine if the introduction of this phase for a biped robot is useful to reduce the energy consumed in the walking. In order to study the efficiency of this phase, six cyclic gaits are presented. The first three cyclic motions are composed of successive single support phases with flat stance foot on the ground separated by double support phases. In the first gait the double support phase is instantaneous and impacts are accepted. The second gait requires an impactless motion with instantaneous double support phases. The third gait requires an impactless 
motion with finite time double support phases, the two feet rotate during this phase. The third last cyclic motions are composed of single support phases that include a sub-phase of rotation of the stance foot about the toe. Motions minimizing an integral criterion based on the vector of the square of the actuated torques are defined. Furthermore, some constraints such as actuator performances and limits on the ground reaction forces are taken into account.

Section 2 presents the dynamic model of the biped. Section 3 is devoted to the formulation of the optimization problem for cycling walking, optimal gaits with and without foot rotation are considered. In section 4 optimal gaits are presented. Section 5 gives the conclusions and perspectives.

\section{The Biped Modeling}

\subsection{The biped}

The biped studied walks in a vertical sagittal $x z$ plane. It is composed of a torso and two identical legs. Each leg is composed of two links and a foot. The ankles, the knees and the hips are one-degree-of-freedom rotational ideal (without friction) actuated joints. Vector $q_{r}=\left[q_{0}, q_{1}, q_{2}, q_{3}, q_{4}, q_{5}, q_{6}\right]^{\prime}{ }^{1}$ (figure 1) describes the shape and the orientation of the biped during a single support phase with a rotation of the stance foot about its toe. During the flat-foot sub-phase, $q_{0}=\pi$ and the vector of joint configuration is reduced to $q=$

$\left[q_{1}, q_{2}, q_{3}, q_{4}, q_{5}, q_{6}\right]^{\prime}$ (figure 3). All links are assumed massive and rigid. The torques are grouped into a $6 \times 1$ torque vector $\Gamma=\left[\Gamma_{1}, \Gamma_{2}, \Gamma_{3}, \Gamma_{4}, \Gamma_{5}, \Gamma_{6}\right]^{\prime}$. The torque $\Gamma_{i}$ is applied at joint $q_{i}$ for $1 \leq i \leq 6$.

\subsection{Gaits studied}

The gait is composed of stance phases separated by double support phases. Each stance phase can be composed of a flat foot phase only or two sub-phases: a flat-foot sub-phase and a foot rotation sub-phase. If the double support is assumed instantaneous, the swing foot is horizontal when the swing foot touches the ground. If the swing leg velocity is not null just before the impact, a discontinuity of joint velocities exists (we denote this transition 'impact') and the stance leg foot takes off. If the swing leg velocity is null just before the impact, joint velocities are continuous (we denote this transition 'impactless') and the two feet are on the ground with zero velocity at the transition. If

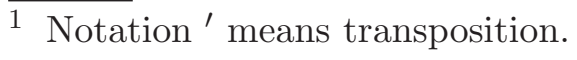




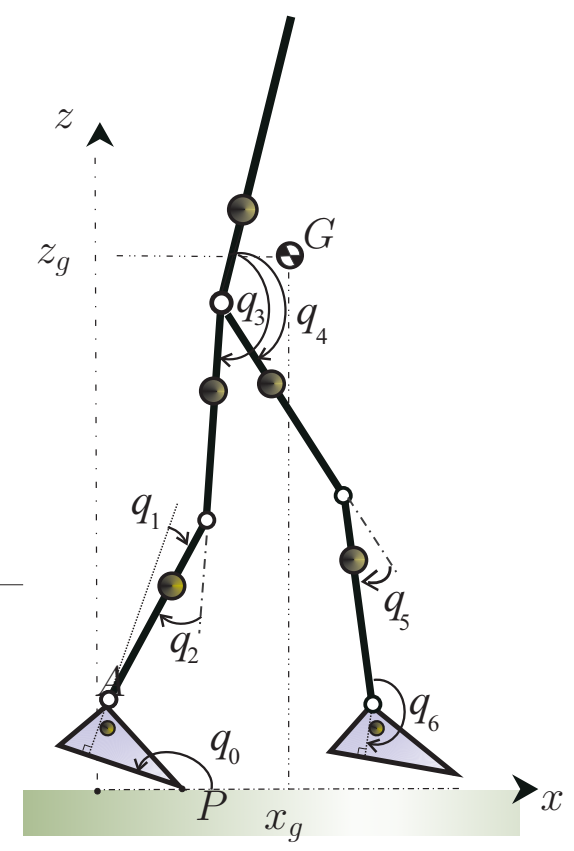

Fig. 1. The studied biped: generalized coordinates

the double support has a finite time duration, the swing foot touches the ground with the heel; the velocity of the heel is null because in other cases the stance leg will take off and it is not possible to have a finite time double support phase (see section 2.3.8). During the double support phase the back foot rotates around its toe, the front foot rotates around its heel until the foot is flat on the ground. In figure 2, the most complex gait is presented, some sub-phases can be suppressed to study simpler gait and to determine the role of each sub-phase on the efficiency of the gait.

In the following, six gait will be studied

- Gait 1: Single supports separated by impact. The supporting foot is always flat on the ground. The swing foot is flat when it touches the ground, the swing foot velocity can be different from zero just before impact.

- Gait 2: Impactless motion composed of successive single support phases. The swing foot is flat when it touches the ground. The impulsive force are null to avoid mechanical stress, the swing foot velocity is null at contact.

- Gait 3: Impactless motion composed of single support and double support phases. In single support, the stance foot is flat on the ground. The swing foot touches the ground with the heel, the linear velocity of the heel is null at contact, in double support the two legs rotate.

- Gait 4: Single supports separated by impacts. The single support is composed of two sub-phases: the supporting foot is flat on the ground and then rotates around the toe. The swing foot is flat when it touches the ground, the swing foot velocity can be different from zero just before impact.

- Gait 5: Impactless motion composed of successive single supports. The single 


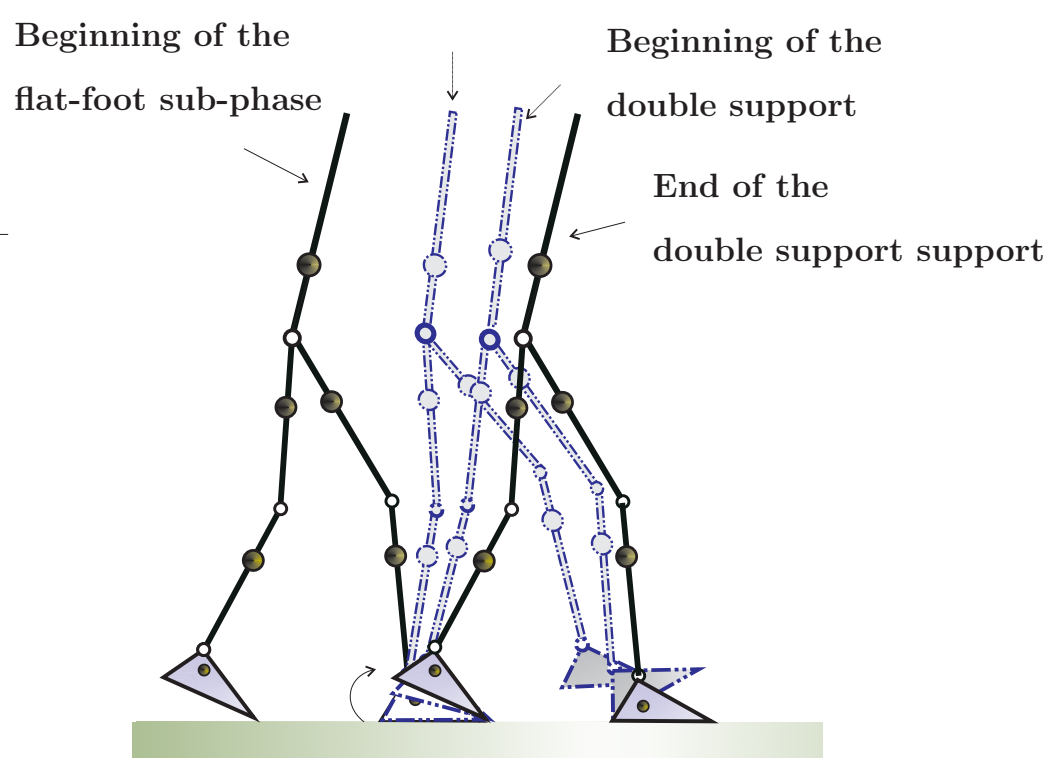

Fig. 2. Decomposition of gait 6 . In single support, at the transition between the two sub-phases, the stance foot of the robot is flat on the ground. The configuration at the end of the single support phase is the configuration at impact, the two feet touch the ground, only the heel of the swing foot touches the ground, only the toe of the stance foot is in contact with the ground. During the double support phase the two feet rotate until the front foot is flat on the ground. The final configuration of double support is the configuration at the beginning of the single support phase with an exchange of legs.

support is composed of two sub-phases: the supporting foot is flat on the ground and then rotates around the toe. The swing foot is flat when it touches the ground, its touchdown velocity is null.

- Gait 6: Impactless motion composed of single support and double support phases. The single support is composed of two sub-phases: the supporting foot is flat on the ground and then rotates around the toe. The swing foot touches the ground with the heel, the linear touchdown velocity of the heel is null at contact, in double support the two feet rotate.

The main characteristics of the gaits are summarized in the table 1 .

\subsection{Dynamic modelling}

The legs swap their roles from one half step to the next one. Thus the study of a half step allows us to deduce the complete behaviour of the robot. The biped dynamic models in support on flat stance foot 1 , with rotation about the toe of the stance foot 1 , the impact model and the double support model 
Table 1

\begin{tabular}{|c|r|r|r|}
\hline gait & single support & double support & transition \\
\hline 1 & flat foot & instantaneous & impact \\
\hline 2 & flat foot & instantaneous & impactless \\
\hline 3 & flat foot & finite time & impactless \\
\hline 4 & 2 sub-phases & instantaneous & impact \\
\hline 5 & 2 sub-phases & instantaneous & impactless \\
\hline 6 & 2 sub-phases & finite time & impactless \\
\hline
\end{tabular}

The 6 studied gaits.

are given.

\subsubsection{The single support phase model: general case}

Using the Lagrange's formalism, the dynamic model can be written, for $k=$ $0, \ldots, 6$ :

$$
\frac{d}{d t}\left(\frac{\partial K}{\partial \dot{q}_{k}}\right)-\frac{\partial K}{\partial q_{k}}+\frac{\partial U}{\partial q_{k}}=Q_{k}
$$

where $K$ is the kinetic energy, $U$ is the potential energy, $Q_{k}$ is the $k^{\text {th }}$ coordinate of the vector of the generalized forces. As the stance leg tip is assumed to be motionless, the virtual work of the reaction force is null.

Thus, the dynamic model can be written as :

$$
M_{r}(q) \ddot{q}_{r}+h_{r}\left(q_{r}, \dot{q}_{r}\right)=\left[\begin{array}{l}
0 \\
\Gamma
\end{array}\right],
$$

where $M_{r}(q)$ is the $(7 \times 7)$-symmetric positive inertia matrix. Let us note as the kinetic energy of the biped is invariant under a rotation of the world frame (19), and as $q_{0}$ defines the orientation of the biped, this matrix is independent of $q_{0}$. Vector $h_{r}\left(q_{r}, \dot{q}_{r}\right)$ contains the centrifugal, Coriolis and gravity forces.

The six last lines of the model can be used to define the torques. The first line describes the behaviour of the robot caused by the unactuated rotation about the toe. 


\subsubsection{The single support phase model: flat-foot sub-phase}

The foot is flat on the horizontal ground thus the variable $q_{0}$ is $\pi$. The configuration of the robot can be described by $q_{r}$ or $q$. This sub-phase exists under the assumption that the zero moment point (ZMP) remains inside the convex hull of the foot support region.

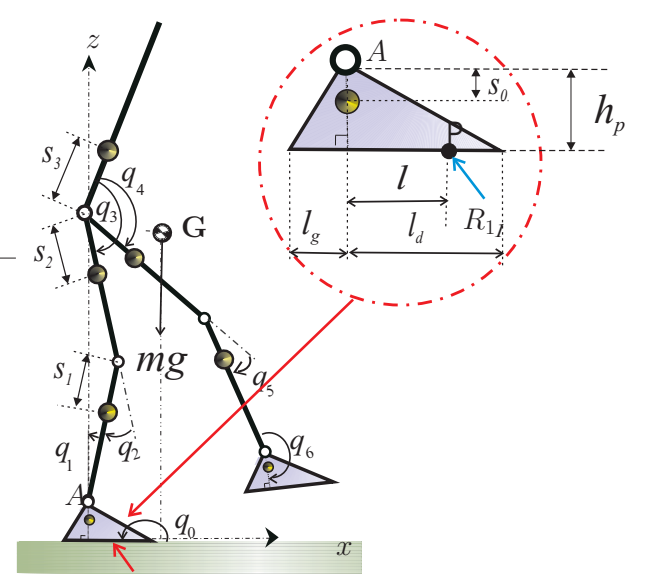

Fig. 3. The flat-foot sub-phase

The dynamic model (2) can be used, it can also be reduced to :

$$
M(q) \ddot{q}+h(q, \dot{q})=\Gamma
$$

where $M(q)$ is the $(6 \times 6)$ low right sub-matrix of $M_{r}$ and the vector $h(q, \dot{q})$ contains the centrifugal, Coriolis and gravity forces, it is composed of the last 6 components of $h_{r}$.

\subsubsection{Reaction force during the single support phase}

During single support, the position of the centre of mass of the biped can be expressed as a function of the angular coordinates, denoted $x_{g}\left(q_{r}\right), z_{g}\left(q_{r}\right)$. When foot- 1 is on the ground, a ground reaction force $R_{1}$ exists. The global equilibrium in translation of the robot makes it possible to calculate this force. The external forces are gravity and the ground reaction force $R_{1}$

$$
m\left[\begin{array}{c}
\ddot{x}_{g} \\
\ddot{z}_{g}
\end{array}\right]+m g\left[\begin{array}{l}
0 \\
1
\end{array}\right]=R_{1} .
$$


where $g$ is the gravity acceleration $m$ is the mass of the biped. Equation (4) can also be written as

$$
\begin{aligned}
m \frac{\partial x_{g}\left(q_{r}\right)}{\partial q_{r}} \ddot{q}_{r}+m \dot{q}_{r}^{\prime} \frac{\partial^{2} x_{g}\left(q_{r}\right)}{\partial q_{r}^{2}} \dot{q}_{r} & =R_{1 x} \\
m \frac{\partial z_{g}\left(q_{r}\right)}{\partial q_{r}} \ddot{q}_{r}+m \dot{q}_{r}^{\prime} \frac{\partial^{2} z_{g}\left(q_{r}\right)}{\partial q_{r}^{2}} \dot{q}_{r}+m g & =R_{1 z},
\end{aligned}
$$

where $\frac{\partial x_{g}\left(q_{r}\right)}{\partial q_{r}}$ and $\frac{\partial z_{g}\left(q_{r}\right)}{\partial q_{r}}$ are $(1 \times 7)$ vectors and $\frac{\partial^{2} x_{g}\left(q_{r}\right)}{\partial q_{r}^{2}}$ and $\frac{\partial^{2} z_{g}\left(q_{r}\right)}{\partial q_{r}^{2}}$ are $(7 \times 7)$ matrices.

The reaction force exerted by the ground must be directed upward to avoid take-off, and the reaction force must be inside the friction cone to avoid the sliding of the biped. These conditions can be written at each time by:

$$
\begin{aligned}
& R_{1 x}+\mu R_{1 z}>0 \\
& -R_{1 x}+\mu R_{1 z}>0
\end{aligned}
$$

where $\mu$ is the friction coefficient (positive). It follows from these two inequalities that $R_{1 z}>0$.

\subsubsection{Flat-foot sub-phase, the ZMP condition}

The equilibrium of the stance foot, in rotation about the ankle allows to determine where the ground reaction force is applied. This reaction force is applied on the sole $(z=0)$, at the abscissa $l$. The external force acting on the stance foot are: its weight, the reaction force exerted by the ground $R_{1}$, the actuated torque $\Gamma_{1}$, the reaction force exerted by the shin on the foot at the ankle.

The equilibrium in rotation about the ankle gives :

$$
0=\Gamma_{1}+m_{0} g x_{g 0}-l R_{1 z}-h_{p} R_{1 x}
$$

where $m_{0}$ is the mass of the foot, $x_{g 0}$ is the $x$-coordinate of the centre of mass of the foot.

The following equation gives the position of the ZMP:

$$
l=\frac{\Gamma_{1}+m_{0} g x_{g 0}-h_{p} R_{1 x}}{R_{1 z}}
$$


The flat foot phase exists only if the foot does not rotate, then if:

$$
-l_{g}<\frac{\Gamma_{1}+m_{0} g x_{g 0}-h_{p} R_{1 x}}{R_{1 z}}<l_{d}
$$

where $l_{g}$ and $l_{d}$ are defined in figure 3 .

\subsubsection{Foot rotation sub-phase, angular momentum about the toe}

The kinetic energy $K$ is independent of the chosen coordinate frame. Since coordinate $q_{0}$ defines only the orientation of the biped as a rigid body, the inertia matrix is independent of this variable. The first equation of system (2) is:

$$
\frac{d}{d t}\left(\frac{\partial K}{\partial \dot{q}_{0}}\right)+\frac{\partial U}{\partial q_{0}}=0
$$

For our planar biped and our choice of coordinates in the single support phase, the term $\frac{\partial K}{\partial \dot{q}_{0}}$ is the biped angular momentum about the stance leg tip $P$ (figure $1)$. We denote this term by $\sigma$. Thus we have:

$$
\frac{\partial K}{\partial \dot{q}_{0}}=\sigma=M_{1} \dot{q}_{r}
$$

where $M_{1}$ is the first line of the inertia matrix $M_{r}$.

The expression $\frac{\partial U}{\partial q_{0}}$ is equal to $-m g\left(x_{g}-l_{d}\right)$. Thus the first equation of the dynamic model of the biped with foot rotation can be written in the following simple form:

$$
\dot{\sigma}=M_{1}\left(q_{r}\right) \ddot{q}_{r}+\dot{q}_{r}^{\prime} \frac{\partial M_{1}}{\partial q_{r}} \dot{q}_{r}=m g\left(x_{g}-l_{d}\right) .
$$

This equation comes from the fact that during this sub-phase the position of the ZMP is imposed, the ZMP is at the toe of the stance foot, and the robot rotates freely around the ZMP. This equation will be used to solve the under-actuation that appears in the dynamic model for this phase: 7 variables $q_{r}$ describe the biped configuration, and only 6 torques are applied.

\subsubsection{The non-instantaneous double support phase}

During the double support phase the back foot rotates around its toe, the front foot rotates around its heel until the foot is flat on the ground. The ground 
exerts a force on the back toe and on the front heel. The dynamic model can be based on the dynamic model (2) when the contact on the front heel is added. The position of the front heel is fixed, the linear velocity and acceleration of the front heel are null. The configuration of the robot can be described by only 5 independent coordinates from $q_{r}$. Its velocities and accelerations variables are such that:

$$
\begin{aligned}
J_{h} \dot{q}_{r} & =0_{2,1} \\
J_{h} \ddot{q}_{r}+h_{h}\left(q_{r}, \dot{q}_{r}\right) & =0_{2,1},
\end{aligned}
$$

where $J_{h}$ is the Jacobian defining the front heel velocity, and the dynamic model is:

$$
M_{r}\left(q_{r}\right) \ddot{q}_{r}+h_{r}\left(q_{r}, \dot{q}_{r}\right)=\left[\begin{array}{c}
0 \\
\Gamma
\end{array}\right]+J_{h}^{\prime} R_{2},
$$

where $R_{2}$ is the force exerted by the ground in the front heel : $R_{2}=\left[R_{2 x}, R_{2 z}\right]^{\prime}$. Since equation (13) is composed of 7 scalar equations and has 8 unknown variables, i.e. 6 components for $\Gamma$ and and 2 components for $R_{2}$, there is an infinite number of forces $R_{2}$ and torques $\Gamma$ that produce the same motion of the robot. On an horizontal ground, any repartition of the horizontal forces such that $R_{1 x}+R_{2 x}=c s t$ will produce the same motion of the robot. Thus the set of solution, $\Gamma, R_{1}, R_{2}$ can be parameterized by $R_{2 x}$. If $J_{h i}$ denotes the $i^{t h}$ line of $J_{h}, \Gamma$ and $R_{2 z}$ are then calculated by:

$$
\left[\begin{array}{c}
\Gamma \\
R_{2 z}
\end{array}\right]=\left[\begin{array}{c|c}
0_{1,6} \\
I_{6}
\end{array} J_{h 2}^{\prime}\right]^{-1}\left(M_{r} \ddot{q}_{r}+h_{r}-J_{h 1}^{\prime} R_{2 x}\right),
$$

Due to the expression of $J_{h}$, the first component of $J_{h 2} \neq 0$ and the matrix used in (14) is invertible.

\subsubsection{Reaction forces during the double support phase}

As for the single support, the global equilibrium in translation of the robot makes it possible to calculate the reaction force on foot 1 . Since the two feet are in support, we have:

$$
m\left[\begin{array}{c}
\ddot{x}_{g} \\
\ddot{z}_{g}
\end{array}\right]+m g\left[\begin{array}{l}
0 \\
1
\end{array}\right]=R_{1}+R_{2} .
$$


Equation (15) can also be written as

$$
\begin{aligned}
& R_{1 x}=m \frac{\partial x_{g}\left(q_{r}\right)}{\partial q_{r}} \ddot{q}_{r}+m \dot{q}_{r}^{\prime} \frac{\partial^{2} x_{g}\left(q_{r}\right)}{\partial q_{r}^{2}} \dot{q}_{r}-R_{2 x} \\
& R_{1 z}=m \frac{\partial z_{g}\left(q_{r}\right)}{\partial q_{r}} \ddot{q}_{r}+m \dot{q}_{r}^{\prime} \frac{\partial^{2} z_{g}\left(q_{r}\right)}{\partial q_{r}^{2}} \dot{q}_{r}+m g-R_{2 z}
\end{aligned}
$$

The reaction force exerted by the ground must be directed upward to avoid take-off, and the reaction force must be inside the friction cone to avoid the sliding of the biped. These conditions must be written independently for both feet:

$$
\begin{aligned}
& R_{1 x}+\mu R_{1 z}>0 \\
& -R_{1 x}+\mu R_{1 z}>0 \\
& R_{2 x}+\mu R_{2 z}>0 \\
& -R_{2 x}+\mu R_{2 z}>0
\end{aligned}
$$

\subsubsection{Impact model}

When the swing foot 2 touches the ground at the end of single support, an impact can occur. We assume that the ground reaction at the impact is described by a Dirac delta-function with intensity $I_{R_{2}}$. This impact is assumed inelastic. This means that the velocity of foot 2 is null just after the impact.

Theoretical two kinds of impact can occur depending on whether the stance foot takes off or not. If the previous stance foot stays on the ground the impulsive force $I_{R_{1}}$ must be upward and included in the friction cone. If the previous stance foot takes off the impulsive force $I_{R_{1}}$ is null and the velocity of the foot after the impact must be directed upward. Numerically for our robot, only the first case (the stance foot takes off) leads to a valid solution. This case produces an instantaneous double support phase. To obtain a non instantaneous double support phase, the velocity of the impacting foot must be null to avoid the impact. This case will be considered in the next sections.

We study some gaits with instantaneous double support phases. This phase is followed with a single support phase where the stance foot is flat on the ground thus we assume that the swing leg impacts the ground with its flat foot. During the impact the stance leg 1 takes off and $I_{R_{1}}=0$ at the instant of impact. The robot configuration $q_{r}$ is assumed to be constant at the instant of double support, and there are jumps in the velocities. The velocity vectors just before and just after impact, are denoted $\dot{q}_{r}^{-}$and $\dot{q}_{r}^{+}$respectively. The torques $\Gamma$ are bounded, thus they do not affect the instantaneous double support. The 
impact model can be written as (8):

$$
\dot{q}_{r}^{+}=\triangle\left(q_{r}\right) \dot{q}_{r}^{-}
$$

where $\triangle\left(q_{r}\right)$ is a $7 \times 7$ matrix. Intensity $I_{R_{2}}$ of the impulsive reaction force is also linear with respect to $\dot{q}_{r}^{-}$:

$$
I_{R_{2}}=I\left(q_{r}\right) \dot{q}_{r}^{-}
$$

Since the previous stance foot takes off, the contact conditions on this leg do not affect the impact equation directly, and the model is independent on the fact that the impact occurs at the end of the flat-foot sub-phase or at the end of the foot rotation sub-phase. The condition of contact of the swing foot at the impact modifies the impact equation, depending if the swing foot touches the ground with the heel or with its flat sole.

The impulsive ground reaction force must be directed upward and be inside the friction cone. To ensure a take-off of leg 2 , the vertical velocity component of leg tip must be positive. In case of a flat-foot impact, the equilibrium of the foot at the impact allows to determine the position of the ZMP at impact for the leg 2. This constraint is developed in (23). All these constraints can be written as some conditions on $\dot{q}_{r}^{-}$.

\section{Gait optimization for the cyclic walking}

\subsection{Principle}

To design a cyclic walking gait, the behaviour of the actuated joint variables are prescribed using a polynomial functions The set of the optimization parameters are used to calculate this polynomial functions, taking account some properties of continuity between the phases. The objective is to calculate a cyclic gait by minimizing a criterion based on the square torques. Physical conditions of contact between the feet and the ground and limits on the actuators define non-linear constraints of this optimization process.

\subsubsection{Polynomial functions}

The biped is driven by six torques, and its configuration is given by coordinates $q$ or $q_{r}$ depending of the phase. To transform the optimization problem into a finite dimension problem, the joint motion is described as a parametric 
function. We choose, for each phase or sub-phase, a polynomial function of time or of a monotonic function.

To insure continuity between two successive phases or sub-phases, the position and velocity of the biped at the beginning and at the end of each phase (or sub-phase) must be taken into account by the parameters of the polynomial functions. So, third-order polynomial functions are needed. Depending of the phase, polynomial functions of order three or four will be used.

\subsubsection{Periodicity condition}

The desired trajectory has the particularity to be cyclic: two successive half steps must be identical and, more precisely, the legs will swap their roles from one half step to the next. The condition of periodicity is used to define the trajectory only on one half step and to reduce the number of optimization parameters. The state of the robot is continuous from one sub-phase or phase to the following one. From the final state of an half-step to the initial state of the following half step, there is an exchange of the number of the joint, since the legs swap their roles, we have:

$$
\begin{aligned}
& q_{1 i}=q_{6 f}-\pi, q_{2 i}=q_{5 f}, q_{3 i}=q_{4 f}, \\
& q_{4 i}=q_{3 f}, \quad q_{5 i}=q_{2 f}, q_{6 i}=q_{1 f}+\pi .
\end{aligned}
$$

In the case of the existence of an impact with no null impulsive force, before the single support phase, a discontinuity of velocity exists. If the state of the robot is known before the impact the state of the robot after the impact can be deduced.

As a consequence these conditions of continuity are used to reduce the number of optimization variables, for each phase the final state of the phase or subphase are optimization variables, but not the initial state, this initial state is deduced from the final state of the previous phase or sub-phase.

Since the stance foot is always in contact with the ground by the toe, any configuration can be represented by $q_{r}$ and there is a maximal number of 7 independent real variables. This number can be reduced in the case of multiple contact with the ground (flat foot or double support). To describe the velocity of the robot, similarly the maximal number of independent real variables is also 7 .

For each phase or sub-phase an optimization parameter is also used to define the duration of the phase or sub-phase. 


\subsection{The different sub-phases}

\subsubsection{The flat foot sub-phase}

The biped is driven by six torques, and its configuration is given in single support phase by six coordinates $q$. To avoid unexpected collision of the swing leg tip with the ground, the joint motion is described by a fourth order polynomials in time:

$$
q_{j}(t)=a_{j 0}+a_{j 1} t+a_{j 2} t^{2}+a_{j 3} t^{3}+a_{j 4} t^{4}, \quad j=1, \ldots, 6
$$

where $j$ is the joint number.

We define an intermediate configuration for the biped at $t=T / 2$. The polynomial functions $q_{j}(t), j=1, \ldots, 6$ are uniquely defined by $q_{i}, q_{f}, q_{i n t}, \dot{q}_{i}, \dot{q}_{f}$. The indices $i, f$ and int correspond to the initial (at $t=0$ ), final (at $t=T$ ) and intermediate (at $t=T / 2$ ) state of the robot, respectively.

Depending on the phase that follows the flat-foot sub-phase, the number of independent variables which describe the final configuration and velocity varies. If the gait is only composed of flat-foot single supports (gaits 1 and 2), the final configuration is a double support configuration with the two feet flat on the ground. Thus only 4 independent variables are necessary to define it. We use the step length $d$, the position of the hip $x_{h}, z_{h}$, and the inclination of the torso $\varphi=q_{1}-q_{2}-q_{3}+\pi$. If this phase is followed by a double support phase (gait 3), the final configuration is a double support configuration with the heel of the front leg on the ground. Thus 5 independent variables are necessary to define it. The angle of the front foot must be added. If this phase is followed by a foot rotation sub-phase (gaits 4 to 6), the final configuration is a single support configuration described by 6 variables.

The final velocity is generally described by 6 variables excepted in the case of an impactless motion without foot rotation in single support (gaits 2 and 3). In these cases the velocity of the swing foot must be such that the impact is avoided. For gait 2, the linear and angular velocities of the swing foot must be null. For gait 3, the linear velocity of the heel must be null.

When function $q(t)$ is chosen, the joint velocity and the joint acceleration can be deduced by the derivation of the polynomial function. The dynamic model (3) gives the torques required to produce the motion. The reaction force can be deduced by (5) with $q_{0}=\pi, \dot{q}_{0}=0, \ddot{q}_{0}=0$. 


\subsubsection{The foot-rotation sub-phase}

During the foot rotation sub-phase, the biped is driven by 6 torques, and its configuration is given by 7 coordinates $q$. Thus the system is under-actuated and its motion cannot be freely chosen. Studies of control of such an underactuated biped (6), (8) have shown that a geometric evolution of the robot $q(s)$ can be chosen. For a given function $q(s)$ within some limits, function $s(t)$ defining a motion compatible with the dynamic model can be deduced using equation (11). In the optimization process, the joint evolution is described by function $q(s)$. This way solves the under-actuation problem and avoids the use of equality constraints as in (7), this point will be detailed.

We choose to define the evolution of the joint variables as a polynomial of $s$, where $s$ is a monotonic function from 0 to 1 . Vectors $q(0), q(1)$ are the initial and final configurations of the foot rotation sub-phase, respectively. Third-order polynomial functions are used:

$$
q_{r j}(s)=b_{j 0}+b_{j 1} s+b_{j 2} s^{2}+b_{j 3} s^{3}, \quad j=0, \ldots, 6
$$

where $j$ is the joint number. The polynomial functions $q_{r j}(s), j=0, \ldots, 6$ are uniquely defined by $q_{i r}, q_{f r}, \frac{d q_{i r}}{d s}, \frac{d q_{f r}}{d s}$. The indices $i r, f r$ correspond to the initial (at $s=0$ ), final (at $s=1$ ) state of the robot for this sub-phase, respectively.

In fact, the initial state for this sub-phase is the final state for the flat-foot rotation sub-phase $q_{i r j}=q_{f j}$ for $j=1, \ldots, 6$ and $q_{i r 0}=\pi$, thus it is known. The initial velocity of the robot is also known, $\dot{q}_{i r j}=\dot{q}_{f j}$ for $j=1, \ldots, 6$ and $\dot{q}_{i r 0}=0$, the initial vector $\frac{d q_{i r}}{d s}$ can be deduced if $\dot{s}(0)$ is known since $\dot{q}_{i r}=\frac{d q_{i r}}{d s} \dot{s}(0)$. The term $\dot{s}(0)$ will be an optimization variable.

The final configuration is a double support configuration with contact on the supporting toe and one foot flat on the ground if there is an instantaneous double support phase; or with contact on the supporting toe and on the heel of the swing foot for finite time double support phase. Thus 5 or 6 coordinates are used to define this configuration.

The final velocity is proportional to $\frac{d q_{f r}}{d s}$. This vector is defined by 7,5 or 4 variables depending if an impact is accepted or not. With an impact, 7 independent velocities variables are necessary to define this vector. For an impactless motion, if the contact with the ground occurs with the heel there are 5 independent velocities variables, and only 4 with a contact on a flat sole. From $\frac{d q_{f r}}{d s}$, the velocity $\dot{q}_{f r}$ can be deduced as it will be seen in the following

\section{From joint trajectories to joint motions}


The joint path is given as $q_{r}(s)$, but since the robot is under-actuated, function $s(t)$ must be such that the biped motion satisfies the dynamic model. Equation (11) can be rewritten as:

$$
\begin{gathered}
\sigma=M_{1}\left(q_{r}\right) \dot{q}_{r}=M_{1}\left(q_{r}\right) \frac{d q_{r}}{d s} \dot{s}=I(s) \dot{s}(s) \\
\dot{\sigma}=m g\left(x_{g}\left(q_{r}(s)\right)-l_{d}\right)
\end{gathered}
$$

These two equations can be combined to have for $0 \leq s \leq 1$ (8):

$$
\begin{aligned}
& \frac{1}{2} I(0)^{2} \dot{s}(0)^{2}=\frac{1}{2} I(s)^{2} \dot{s}(s)^{2}+V(s) \\
& V(s)=-m g \int_{0}^{s} I(\xi)\left(x_{g}(\xi)-l_{d}\right) d \xi
\end{aligned}
$$

Since functions $I(s)$, and $V(s)$ can be calculated for any given function $q_{r}(s)$, it follows that the initial value $\dot{s}(0)$ permits to define the function $\dot{s}(s)$ completely and thus $s(t)$.

$$
\dot{s}(s)=\sqrt{\frac{I(0)^{2} \dot{s}(0)^{2}-2 V(s)}{I(s)^{2}}}
$$

Polynomials $q_{r}(s)$ is defined with the assumption that $s$ is a well defined increasing function, thus the following conditions must be satisfied:

$$
\begin{gathered}
\dot{s}(0)>\sqrt{2 \frac{\max _{s \in[0,1]}(V(s))}{I(0)^{2}}} \\
I(s) \neq 0 \text { for } \quad 0 \leq s \leq 1
\end{gathered}
$$

These constraints are taken into account in the optimization process.

The value of $\dot{s}$ at the end of the foot rotation sub-phase can be deduced from equation (23), thus the velocity of the robot at the end of the foot rotation sub-phase is :

$$
\dot{q}_{f r}=\sqrt{\frac{I(0)^{2} \dot{s}(0)^{2}-2 V(1)}{I(1)^{2}}} \frac{d q_{f r}}{d s}
$$

\section{Torques and forces}

For the foot rotation sub-phase, when the function $q_{r}(s)$ is chosen, $\dot{s}(s)$ can 
be calculated by equation (23). Thus the joint velocity is:

$$
\dot{q}_{r}(s)=\frac{d q_{r}}{d s} \dot{s}(s)
$$

Since the joint acceleration can be written:

$$
\ddot{q}_{r}(s)=\frac{d^{2} q_{r}}{d s^{2}} \dot{s}^{2}+\frac{d q_{r}}{d s} \ddot{s}(s)
$$

The first line of the dynamic model permits to deduce $\ddot{s}$. Then the last six lines give the torques required to produce the motion:

$$
\begin{aligned}
& \ddot{s}(s)=\frac{m g\left(x_{g}\left(q_{r}(s)\right)-l_{d}\right)-M_{1}\left(q_{r}\right) \frac{d^{2} q_{r}}{d s^{2}} \dot{s}^{2}-\dot{q}_{r}^{T} \frac{\partial M_{1}\left(q_{r}\right)}{\partial q_{r}} \dot{q}_{r}}{M_{1}\left(q_{r}\right) \frac{d q_{r}}{d s}} \\
& \Gamma=M_{2,7}\left(q_{r}(s)\right)\left(\frac{d^{2} q_{r}}{d s^{2}} \dot{s}^{2}+\frac{d q}{d s} \ddot{s}(s)\right)+h_{2,7}(q, \dot{q})
\end{aligned}
$$

where $M_{2,7}$ is composed of the last six rows of $M_{r}, h_{2,7}$ is composed of the last six rows of $h_{r}$. The reaction force can be deduced by (5).

\subsubsection{Finite time double support phase}

The biped is driven by six torques, and its configuration is given in double support phase by five independent coordinates only. The configuration is described by 7 coordinates vector $q_{r}$ as in the foot rotation phase but the position of the front heel is constant and linear velocity and acceleration of the heel are null. The joint motion is described by a third order polynomials in time for the joints $0,1,2,3,6$, the evolution of the joints $q_{4}$ and $q_{5}$ are determined in order to satisfy the contact constraint on the front heel (12).

The polynomials function are:

$$
q_{r j}(t)=c_{j 0}+c_{j 1} t+c_{j 2} t^{2}+c_{j 3} t^{3} \quad j=0,1,2,3,6
$$

where $j$ is the joint number. They are uniquely defined by the initial configuration and velocity and the final configuration and velocity. A non-instantaneous double support phase exists only for impactless motion, thus the initial state of the robot is known as the final state of the previous phase.

This phase is followed by a single support on flat stance foot and the swing foot is in contact with the toe. Since one point on each feet is fixed during the double support phase, the step length can not be chosen at the end of the double support phase, the final configuration is determined by 4 independent 
variables: the position of the hip $x_{h}, z_{h}$, the inclination of the torso $q_{1}-q_{2}-$ $q_{3}+\pi$ and the inclination of the back feet.

Only 5 components of the joint velocity are independent during this phase, thus the final velocity can be defined by 5 variables at maximum, but since impactless motion is defined, the angular velocity of the front feet must be null at the end of the double support to avoid impact, thus the final velocity is defined by 4 variables only.

When function $q(t)$ is chosen, the joint velocity and the joint acceleration can be deduced by the derivation of the polynomial function. To determine the torques and reaction forces the dynamic model (13) must be solved. As a consequence the reaction force $R_{2 x}$ must be known. $R_{2 x}$ is defined in the optimization process as a second order polynomial function of time:

$$
R_{2 x}(t)=d_{0}+d_{1} t+d_{2} t^{2}
$$

Then the torques and reaction force are deduced by (14) and (16).

\subsection{Number of optimization variables}

Depending of the gait studied, the number of optimization variables can be deduced from the description of each sub-phases. The results is summarized in table 2 .

\subsection{Statement of the optimization problem}

By parametrizing the joint motion in terms of polynomial functions, the optimization problem reduces to a constrained parameter optimization problem of the form

$$
\begin{aligned}
& \text { Minimize } \mathcal{C}(P) \\
& \text { subject to } g_{j}(P) \leq 0 \text { for } j=1,2, \ldots, l
\end{aligned}
$$

where $P$ is the set of optimization variables described in table $2, \mathcal{C}(P)$ is the criterion to minimize with $l$ inequality constraints $g_{j}(P) \leq 0$ to satisfy. The criterion and constraints are given in the following sections.

For the numerical optimization we use the sequential quadratic programming method (SQP). This local method uses quasi-Newton approximations for the 
Hessian of the augmented Lagrangian and obtains search directions from a sequence of quadratic programming sub-problems. The algorithm fmincon from the package Matlab is used to solve these constrained parameter optimization problem.

\subsubsection{The constraints}

The objective of this study is to define optimal motion for a given robot with given actuators. The constraints on the maximum torques $\left(\Gamma_{\max }\right)$ and velocities $\left(V_{\max }\right)$ of the actuators are considered.

Some other constraints must be checked to insure that the optimal trajectory is convenient:

- constraints on reaction force (section 2.3.3 and 2.3.7)

- constraints on impact phase (section 2.3.8)

- the swing leg foot must not touch the ground before the prescribed end of the stance phase

- constraints on the ZMP for the flat-foot sub-phase (section 2.3.4)

- constraints on the existence of the function $s(t)$ (eq. (24))

Table 2

\begin{tabular}{|c|c|c|c|c|c|c|c|}
\hline \multirow{2}{*}{ sub-phase } & variables & \multicolumn{5}{|c|}{ Gait } \\
& & 1 & 2 & 3 & 4 & 5 & 6 \\
\hline \hline \multirow{2}{*}{ flat foot } & final configuration & 4 & 4 & 5 & 6 & 6 & 6 \\
& final velocity & 6 & 3 & 4 & 6 & 6 & 6 \\
& duration & 1 & 1 & 1 & 1 & 1 & 1 \\
& intermediate & & & & & & \\
& configuration & 6 & 6 & 6 & 6 & 6 & 6 \\
\hline \hline \multirow{2}{*}{ foot } & final configuration & & & & 5 & 5 & 6 \\
rotation & "final velocity" $\frac{d q_{f r}}{d s}$ & & & & 7 & 4 & 5 \\
& initial value $\dot{s}(0)$ & & & & 1 & 1 & 1 \\
\hline \hline \multirow{2}{*}{ double } & final configuration & & & 4 & & & 4 \\
support & final velocity & & & 4 & & & 4 \\
& duration & & & 1 & & & 1 \\
& torque & & & 3 & & & 3 \\
\hline \hline
\end{tabular}

Number of optimization variables 
All these constraints (excepted the last one) that must be satisfied at each instant of the cycle are sampled at 20 points of each phase to produce a discrete set of inequality conditions.

\subsubsection{The criterion}

In electrical motors and for a cycle of walk, most part of the energy consumption is due to the loss by Joule effect neglecting the friction. Thus the optimized criterion is proportional to this loss of energy. It is defined as the integral of the norm of the torques for a displacement of one meter:

$$
\mathcal{C}_{\boldsymbol{I}}=\frac{1}{d} \int_{0}^{T} \Gamma(t)^{\prime} \Gamma(t) d t
$$

where $T$ is the duration of one half step, $d$ is the distance travelled by the centre of mass during one half step. All the sub-phases are taken into account.

This criterion will be optimized for a given motion velocity of the robot $v_{x}$, thus the criterion is rewritten to includes this equality constraint via the penalty method:

$$
\mathcal{C}=\frac{1}{d} \int_{0}^{T} \Gamma(t)^{\prime} \Gamma(t) d t+1 . e^{10}\left(v_{x}-d / T\right)^{2}
$$

\section{Optimal walk}

\subsection{The prototype used for the optimization}

In the presented study, we use the parameters of a humanoid robot with a motion limited to a plane. The main parameters for this robot are given in table 3, the weight and height of this robot are not far from human weight and height (the position of the centre of mass corresponds to the distance $s_{i}$ in figure 3). To avoid take-off of the stance foot, the minimal vertical reaction force is $100 \mathrm{~N}$. The friction coefficient is $2 / 3$. The evolution of the swing leg foot must be higher than a sinusoidal function. The maximum of this function is $0.03 \mathrm{~m}$. The initial orientation of the torso is between -6 and 20 degrees. 
Table 3

\begin{tabular}{|c|r|r|r|r|}
\hline links & feet & femur & tibia & torso \\
\hline length in $m$ & 0.21 & 0.3 & 0.3 & 0.7 \\
\hline mass in $\mathrm{kg}$ & 2.3 & 1.7 & 2 & 44 \\
\hline inertia in $\mathrm{kg} \cdot \mathrm{m}^{2}$ & 0.01 & 0.022 & 0.025 & 2.53 \\
\hline mass centre position in $\mathrm{m}$ & 0.05 & 0.13 & 0.15 & 0.31 \\
\hline \hline motors & ankle & $\mathrm{knee}$ & $\mathrm{hip}$ \\
\hline actuator inertia in $\mathrm{kg} \cdot \mathrm{m}^{2}$ & 0. & 0. & 0. \\
\hline Torque max in $\mathrm{Nm}$ & 130 & 170 & 150 \\
\hline Vit max in $\mathrm{rd} \cdot \mathrm{s}^{-1}$ & 5 & 4 & 4.4 \\
\hline \hline feet characteristics & $h_{p}$ & $l_{d}$ & $l_{g}$ \\
\hline in $\mathrm{m}$ & 0.1 & 0.12 & 0.09 \\
\hline
\end{tabular}

Parameters for optimization.

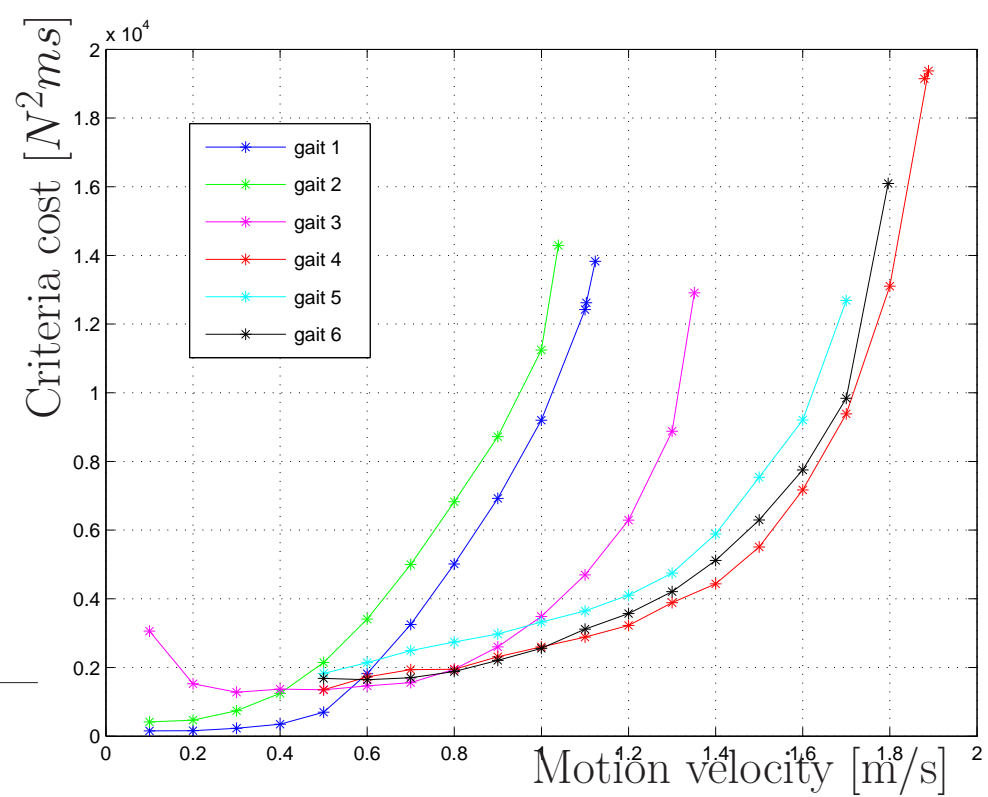

Fig. 4. The cost criteria as function of the motion velocity for the six different gaits studied.

\subsection{Walk at various velocities}

In figure 4, the evolution of the cost criteria are drawn as function of the motion velocity for the six gaits studied (see section 2.2). For slow motion (the average velocity is less than $0.58 \mathrm{~m} / \mathrm{s}=2 \mathrm{~km} / \mathrm{h}$ ), walk composed of flat foot single support phase separated by instantaneous double support phases (gait 1) is the most efficient walk with respect to the criterion studied. For intermediate 
velocity (from $0.58 \mathrm{~m} / \mathrm{s}=2 \mathrm{~km} / \mathrm{h}$ to $0.8 \mathrm{~m} / \mathrm{s}=2.88 \mathrm{~km} / \mathrm{h}$ ), a walk with flatfoot single support and non instantaneous double support (gait 3 ) is the best gait. For fast walking (from $0.8 \mathrm{~m} / \mathrm{s}=2.88 \mathrm{~km} / \mathrm{h}$ to $1.8 \mathrm{~m} / \mathrm{s}=6.48 \mathrm{~km} / \mathrm{h}$ ), the best gaits (gait 4 and 6 ) include a rotation of the stance phase in single support, an impact or an impactless motion with double support phase. The difference between gait 1 and 2, and gait 4 and 5, is only the existence of an no null impulsive reaction force in the impact phase, the evolutions of the criteria are similar but the avoidance of the impulsive reaction force sightly increases the criterion cost. With the same actuators the maximal motion velocity is dramatically increased by the introduction of the foot rotation sub-phase.

\subsection{Walk with Gait 1}

For a motion velocity of $0.5 \mathrm{~m} / \mathrm{s}(1.8 \mathrm{~km} / \mathrm{h})$, gait 1 is the best gait. The optimal walk has the following characteristics: for one half step, the duration $\mathrm{T}$ is $0.46 \mathrm{~s}$, the step length is $0.23 \mathrm{~m}$. The value of the torque cost criterion $\mathcal{C}$ is $697 N^{2} \mathrm{~ms}$.

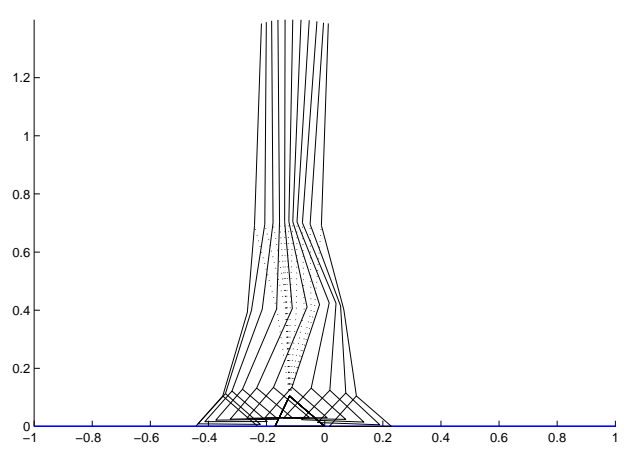

Fig. 5. Stick diagram for gait 1 at $0.5 \mathrm{~m} / \mathrm{s}$.

Figure 5 presents the stick-diagram of one step of optimal walk. Figure 6 regroups the torques after the gear box, the reaction force, the evolution of the ZMP as function of the time. The limits on the torques are satisfied, the maximal value for the actuator are given in table 3 . The conditions to avoid take-off and sliding are satisfied. The torques at the beginning and the end of the step are higher than at the middle of the step, these phases allow to accelerate and decelerate the robot. For this slow gait it can be noticed that the ZMP evolves close to the vertical of the ankle, because the torque at ankle is low. 

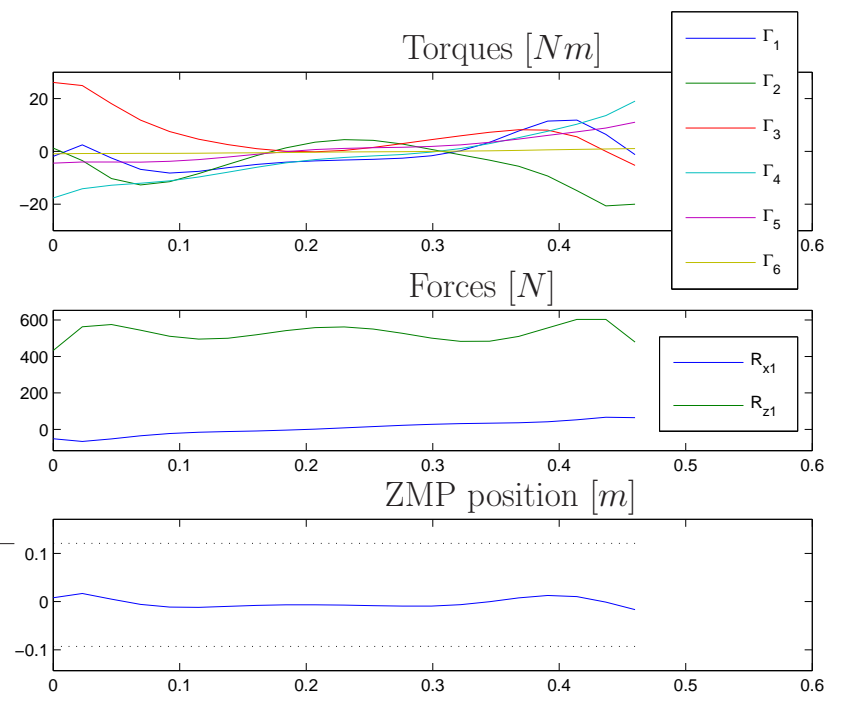

Fig. 6. The optimal motion with gait 1 at $0.5 \mathrm{~m} / \mathrm{s}$ : torques, forces and evolution of the ZMP

\subsection{Walk with Gait 2}

For a motion velocity of $0.4 \mathrm{~m} / \mathrm{s}(1.44 \mathrm{~km} / \mathrm{h})$, the gait 2 can also be used. The criterion cost is higher that for the gait 1 because the avoidance of the impact increases the torque needed during the motion. The optimal walk with gait 2 has the following characteristics: for one half step, the duration $\mathrm{T}$ is $0.48 \mathrm{~s}$, the step length is $0.191 \mathrm{~m}$. The value of the torque cost criterion $\mathcal{C}$ is $1247 N^{2} \mathrm{~ms}$.

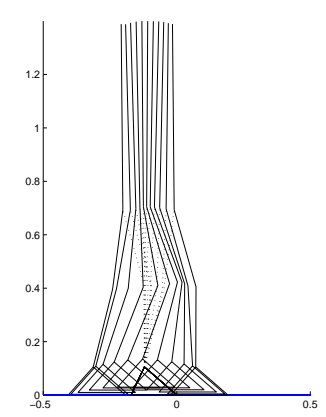

Fig. 7. Stick diagram for gait 2 at $0.4 \mathrm{~m} / \mathrm{s}$.

Figure 7 presents the stick-diagram of one step of optimal walk. Figure 8 regroups the torques, the reaction forces, the evolution of the ZMP as function of the time. The conditions to avoid take-off and sliding are satisfied. For this slow gait, the ZMP evolves close to the vertical of the ankle, because the torque at ankle is low. Since the torques at the stance ankle are higher than for the gait 1, the ZMP moves from the vertical of the ankle. 

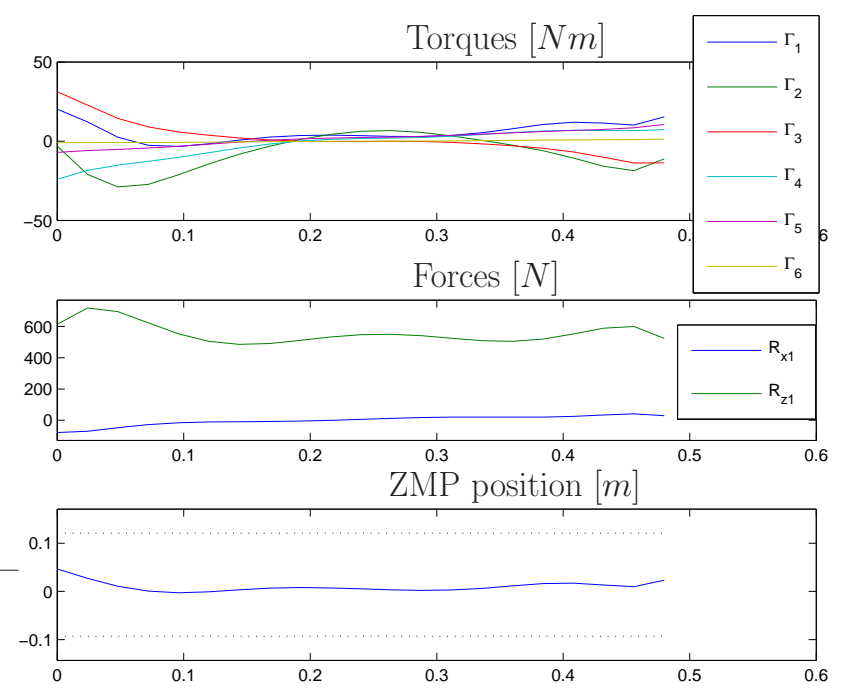

Fig. 8. The optimal motion for gait 2 at $0.4 \mathrm{~m} / \mathrm{s}$ : torques, forces, and evolution of the ZMP.

\subsection{Walk with Gait 3}

For a motion velocity of $0.7 \mathrm{~m} / \mathrm{s}(2.5 \mathrm{~km} / \mathrm{h})$, gait 3 is the best gait. The optimal walk includes a non instantaneous double support phase, it has the following characteristics: for one half step, the duration $\mathrm{T}$ is $0.563 \mathrm{~s}$, the step length is $0.394 \mathrm{~m}$. The double support phase has a duration that represents $20 \%$ of the duration of the step. The value of the torque cost criterion $\mathcal{C}$ is $1557 \mathrm{~N}^{2} \mathrm{~ms}$. The duration and the step length have increases with respect to the previous gait.

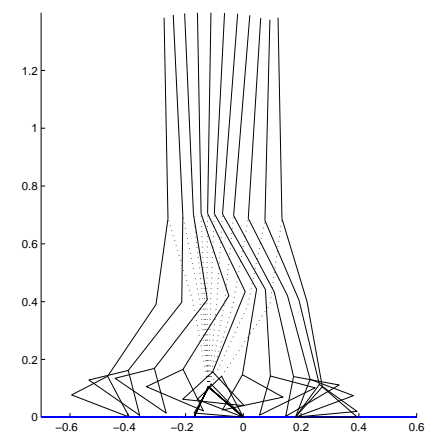

Fig. 9. Stick diagram for gait 3 at $0.7 \mathrm{~m} / \mathrm{s}$.

Figure 9 presents the stick-diagram of one step of optimal walk. During the double support phase the two feet rotate, thus the back foot is not flat on the ground at the beginning of the single support phase. Figure 10 regroups the torques, the reaction forces, the evolution of the ZMP as function of the time. The limits on the torques are satisfied, the torque are higher in double support phase than in single support phase. For this gait it can be noticed that the ZMP evolves close to the vertical of the ankle, because the torque at ankle is 

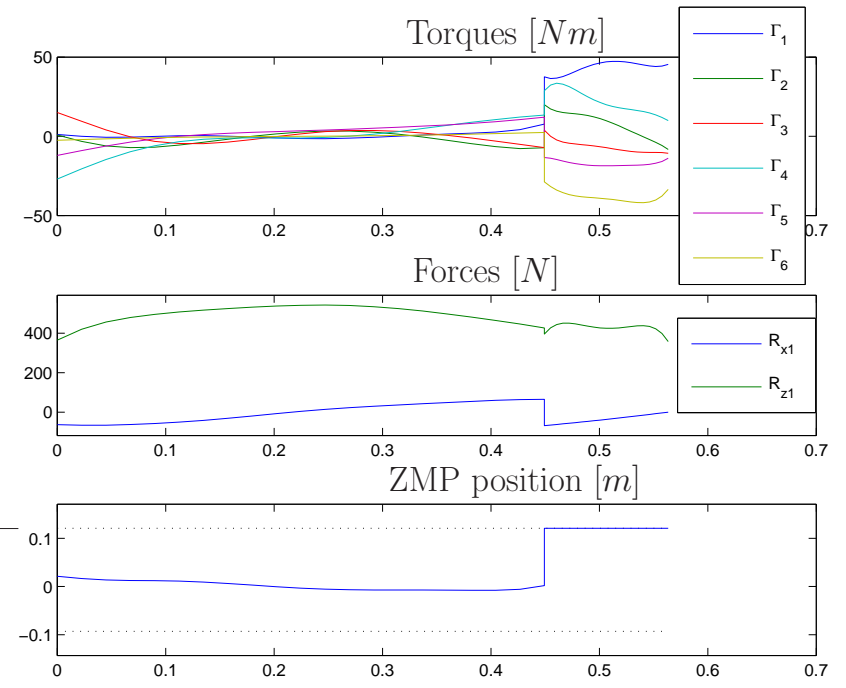

Fig. 10. The optimal motion for gait 2 at $0.7 \mathrm{~m} / \mathrm{s}$ : torques, forces and evolution of the ZMP.

low in single support phase. In double support phase, only the ZMP for the back foot is presented, since the foot rotates around the toe, the ZMP is at the front limit of the foot. For the front foot, since the foot rotates around the heel, the ZMP is at the back limit of the foot.

\subsection{Walk with Gait 4}

For a motion velocity of $1.2 \mathrm{~m} / \mathrm{s}(4.32 \mathrm{~km} / \mathrm{h})$, gait 4 is the best gait. The optimal walk includes a foot rotation in the single support phase, it has the following characteristics: for one half step, the duration $\mathrm{T}$ is $0.28 \mathrm{~s}$, the step length is $0.336 \mathrm{~m}$. The duration of the first sub-phase with stance flat foot is $0.112 s$ and the duration of the stance foot-rotation sub-phase is $0.147 \mathrm{~s}$. The value of the torque cost criterion $\mathcal{C}$ is $3224 N^{2} \mathrm{~ms}$.

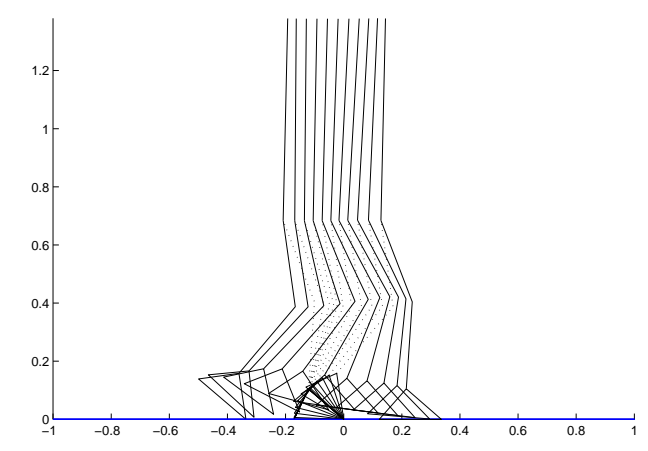

Fig. 11. Stick diagramfor gait 4 at $1.2 \mathrm{~m} / \mathrm{s}$.

Figure 11 presents the stick-diagram of one step of optimal walk. During the second simple support sub-phase the stance foot rotates about its toe, thus 

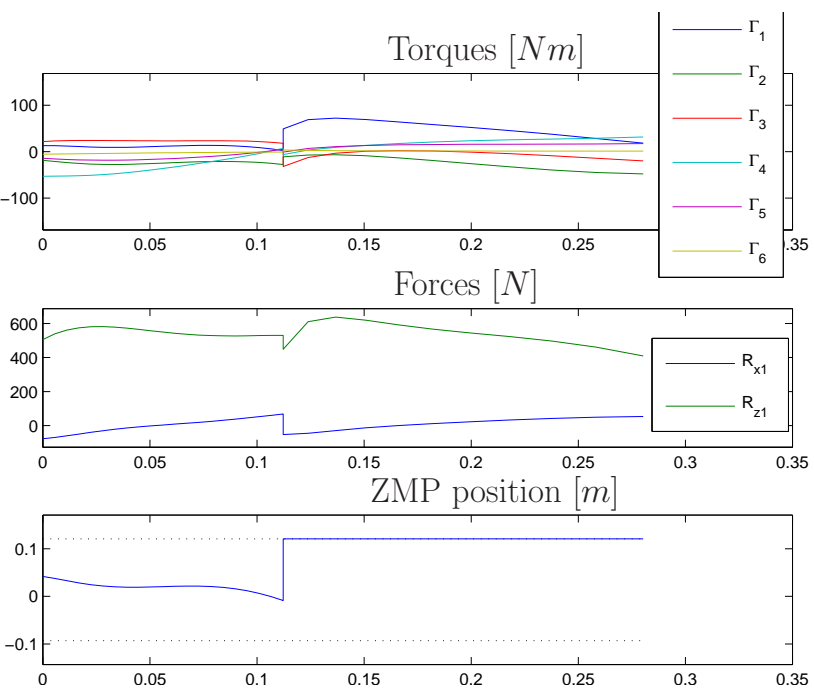

Fig. 12. The optimal motion with gait 4 at $1.2 \mathrm{~m} / \mathrm{s}$ : torques, forces and evolution of the ZMP

the back foot is not flat on the ground at the beginning of the single support phase. Figure 12 regroups the torques after the gear box, the reaction force, the evolution of the ZMP as function of the time. The limits on the torques are satisfied, the torque are higher in the foot rotation sub-phase than in the flat foot sub-phase. In foot-rotation sub-phase, since the foot rotates around the toe, the ZMP is at the front limit of the size of the feet.

\subsection{Walk with Gait 5}

For the motion velocity of $1.2 \mathrm{~m} / \mathrm{s}$, the gait 5 can be used. The optimal walk has the following characteristics: for one half step, the duration $\mathrm{T}$ is $0.317 \mathrm{~s}$, the step length is $0.381 \mathrm{~m}$. The duration of the first sub-phase with stance flat foot is $0.171 s$ and the duration of the stance foot-rotation sub-phase is 0.146 $s$. The value of the torque cost criterion $\mathcal{C}$ is $4105 \mathrm{~N}^{2} \mathrm{~ms}$.

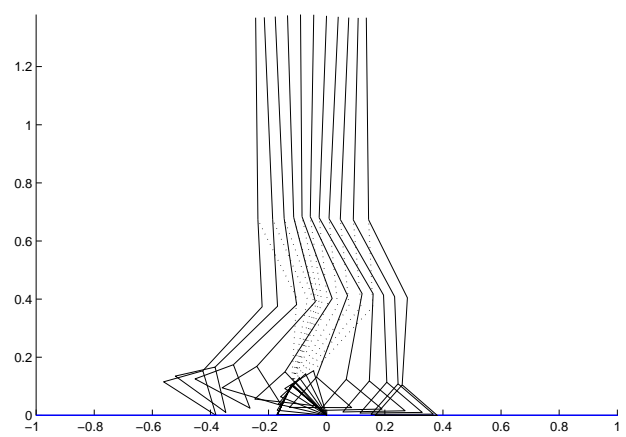

Fig. 13. Stick diagram for gait 5 at $1.2 \mathrm{~m} / \mathrm{s}$.

Figure 13 presents the stick-diagram of one step of optimal walk. Figure 14 

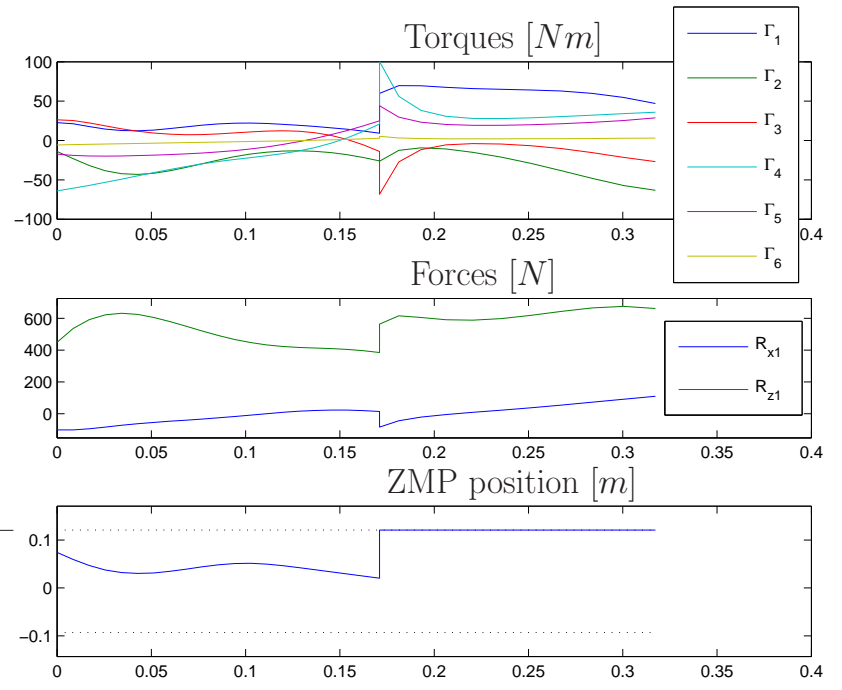

Fig. 14. The optimal motion with gait 5 at $1.2 \mathrm{~m} / \mathrm{s}$ : torques, forces and evolution of the ZMP.

regroups the torques, the reaction forces, the evolution of the ZMP as function of the time. The torques at the beginning of the step are higher than for gait 4 , as a consequence the ZMP moves from the vertical axis of the ankle.

\subsection{Walk with Gait 6}

For a motion velocity of $1.2 \mathrm{~m} / \mathrm{s}(4.32 \mathrm{~km} / \mathrm{h})$, gait 6 is a good gait, this gait is the closest to human gait. The optimal walk includes a foot rotation in the single support phase and a double support phase. With respect to gait 4 , this impactless motion reduces the mechanical stress in the robot. It has the following characteristics: for one half step, the duration $\mathrm{T}$ is $0.384 \mathrm{~s}$, the step length is $0.460 \mathrm{~m}$. The duration of the first sub-phase with stance flat foot is $0.170 \mathrm{~s}$, the duration of the stance foot-rotation sub-phase is $0.164 \mathrm{~s}$ and the duration of the double support phase is $0.050 \mathrm{~s}$ (this duration is the minimal value accepted for a double support phase). The value of the torque cost criterion $\mathcal{C}$ is $3570 \mathrm{~N}^{2} \mathrm{~ms}$.

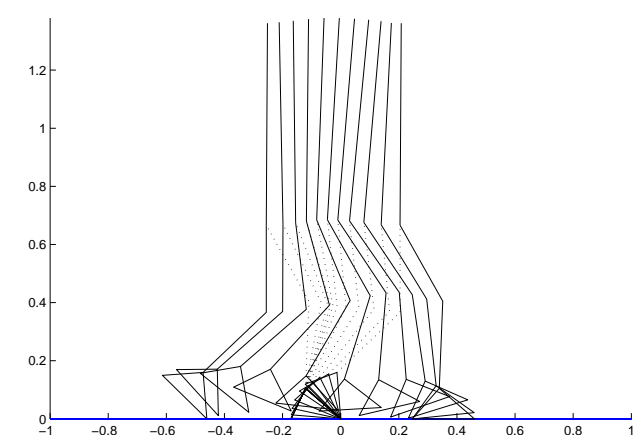

Fig. 15. Stick diagram for gait 6 at $1.2 \mathrm{~m} / \mathrm{s}$. 

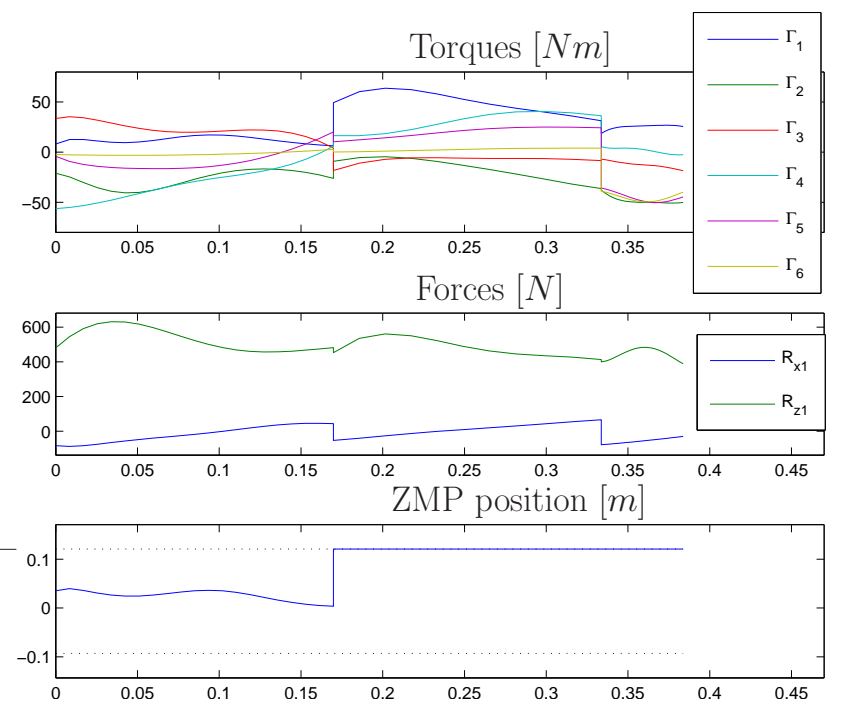

Fig. 16. The optimal motion with gait 6 at $1.2 \mathrm{~m} / \mathrm{s}$ : torques, forces and evolution of the ZMP.

Figure 15 presents the stick-diagram of one step of optimal walk. During the second simple support sub-phase the stance feet rotates about its toe. This rotation continue during the double support phase. During this last phase the front foot rotates also about the heel to be flat on the floor at the end of this phase. Figure 16 regroups the torques, the reaction forces, the evolution of the ZMP as function of the time. In foot-rotation sub-phase and in the double support phase, since the foot rotates around the toe, the ZMP is at the front limit of the feet.

\subsection{Optimal Gaits for various bipeds}

Three different bipeds have been studied in order to define if the optimal gait depends on the robot or not. Three experimental bipeds are considered to define the various physical parameters sets. The heaviest biped has been considered in section 4.2. The second biped is developed by French laboratories in the framework of the Phema project. The bipedal robot is an humanoid that can move in three dimensional space but this study is limited to planar motion only. The biped feet are composed of two link articulated by a rotational joint to study motion with rotation of the feet. In the modelling only the first link of the feet is considered. The third biped considered is Rabbit, this planar bipedal robot has been defined to be able to run and to have fast motion. This French biped (6) has been developed in a national project, it has point contact with the ground but for this study feet have been added. This bipedal robot is the lightest, the weight of the legs are not negligible with respect to the mass of the torso. The characteristics of the bipedal robots are given in table 4 . 


\begin{tabular}{|c|r|r|r|r||r|r|r|r|}
\hline links & feet & femur & tibia & torso & feet & femur & tibia & torso \\
\hline length in $m$ & 0.20 & 0.39 & 0.39 & 0.4 & 0.21 & 0.4 & 0.4 & 0.62 \\
\hline mass in $\mathrm{kg}$ & 0.68 & 2.19 & 5 & 25 & 1 & 3.2 & 6.8 & 17 \\
\hline inertia in $\mathrm{kg} \cdot \mathrm{m}^{2}$ & 0.002 & 0.027 & 0.066 & 1.03 & 0.012 & 0.048 & 0.069 & 1.89 \\
\hline mass centre pos. $[\mathrm{m}]$ & 0.02 & 0.22 & 0.22 & 0.28 & 0.03 & 0.13 & 0.16 & 0.14 \\
\hline \hline \multicolumn{2}{|c|}{ motors } & ankle & knee & hip & ankle & knee & hip \\
\hline actuator inertia in $\mathrm{kg} \cdot \mathrm{m}^{2}$ & 0. & 0. & 0. & 0. & 0.83 & 0.83 \\
\hline Torque max in $\mathrm{Nm}$ & 157 & 108 & 150 & 150 & 150 & 150 \\
\hline Vit max in $\mathrm{rd} \cdot \mathrm{s}^{-1}$ & 4.3 & 7 & 2.7 & 4.2 & 8.4 & 8.4 \\
\hline \hline feet caracteristics & $h_{p}$ & $l_{d}$ & $l_{g}$ & $h_{p}$ & $l_{d}$ & \multicolumn{2}{l|}{$l_{g}$} \\
\hline in $m$ & 0.06 & 0.13 & 0.07 & 0.08 & 0.2 & 0.05 \\
\hline
\end{tabular}

Table 4

Parameters for the robot Phema with planar motion and for the robot Rabbit with feet

The cost criteria for the different gaits are drawn as function of the joint velocity for the biped Phema and Rabbit in figure 17 and figure 18 respectively.

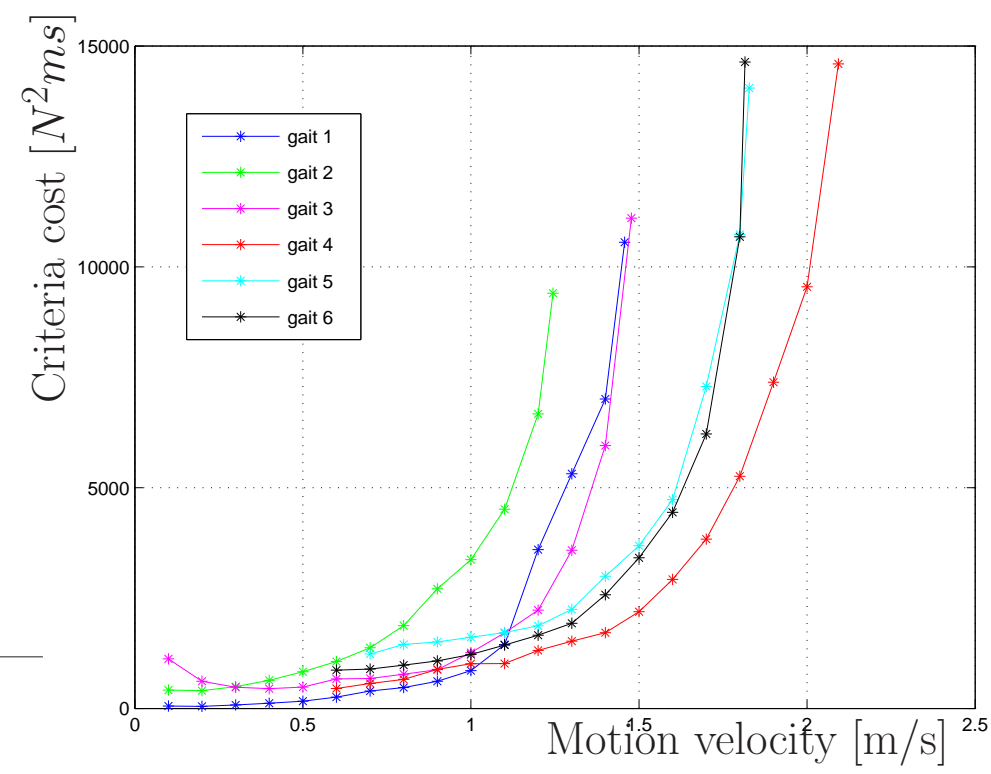

Fig. 17. The cost criteria in $\mathrm{N}^{2} \mathrm{~ms}$ as function of the motion velocity in $\mathrm{m} / \mathrm{s}$ for the six different gaits studied for robot Phema with planar motion.

For the three robots studied, we can observe that for fast motion the best gaits include a foot rotation sub-phase, for a slow motion this sub-phase is not useful.

For each group of gaits with foot rotation (gaits 456 ), the gait with the 


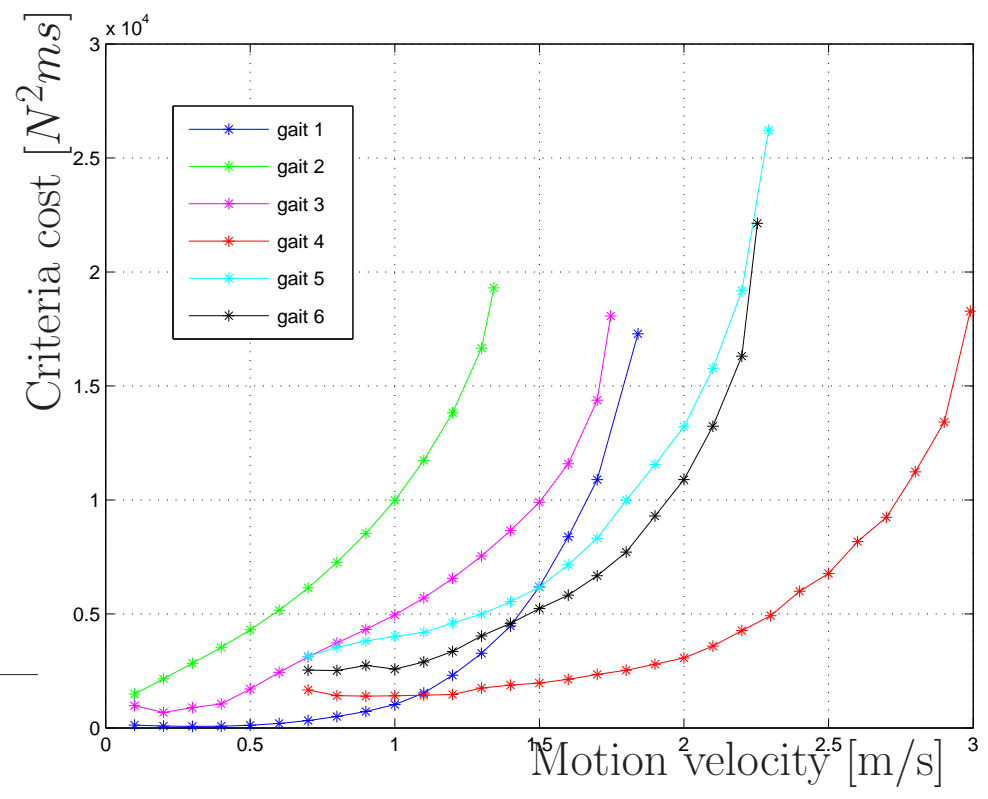

Fig. 18. The cost criteria in $\mathrm{N}^{2} \mathrm{~ms}$ as function of the motion velocity in $\mathrm{m} / \mathrm{s}$ for the six different gaits studied for robot Rabbit with feet.

lowest cost includes impact phases. If the impact must be avoided to preserve the biped's mechanics then it is slightly better to introduce a double support phase. In the optimisation process, the minimal duration of the double support phase is $0.05 \mathrm{~s}$, for fast motion, this double support phase can be suppressed.

For gaits without foot rotation or double support (gaits 1,3), the gait with impact has a lower cost that the gait without impact. One of the main difference that can be observed for the different bipeds is the efficient of the gait with double support and without foot rotation (gait 2), it can be the best gait for an intermediate velocity or never a better gait than gait 1.

Depending of the robot characteristics, the fastest motion velocity obtained with the introduction of the foot rotation can be $30 \%$ to $100 \%$ higher than for a motion without foot rotation in single support.

\section{Conclusions}

Optimal cyclic joint reference trajectories for the cyclic walking of a biped were proposed in this paper. Classical algebraic optimization technique is used, the optimal trajectory is defined by a reduced number of parameters (14 to 43). Some inequality constraints such as the limits on torques and joint velocities, the conditions of no take-off and no sliding during motion and impact, the constraints on the ZMP, some limits on the motion of the free leg are taken into account. The optimized walking gait is composed of single sup- 
ports and instantaneous double supports defined by passive impacts or non instantaneous double supports with rotation of the back foot about its toe and rotation of the front foot about its heel. The single support phase can include a foot-rotation sub-phase or not. The optimization process is able to deal with under-actuated and over-actuated sub-phases.

Six gaits are explored. Optimal motion are presented for each gait. This study includes the evolution of the criterion cost for the six gaits with respect to the different motion velocities, and for physical parameters of three experimental bipeds with different mass repartition and actuator limits. We observe:

- With the same actuators the maximal motion velocity is dramatically increases by the introduction of the foot rotation sub-phase.

- The foot-rotation sub-phase in single support allows to reduce significantly the cost criterion for fast motions.

The next step is to extend this study to the cases of a walking gait in 3D.

\section{References}

[1] H. Beaupied. Étude mécanique et énergétique de la marche, de la course et de la transition marche-course: influence de la spécialité athlétique. Phd thesis, Rennes II, France, October 2003.

[2] V.V. Beletskii, V.E. Berbyuk, and V.A. Samsonov. Parametric optimization of motions of a bipedal walking robot. Izv An SSSR Mekhanika Tverdogo Tela [Mechanics of Solids], 17(1):28-40, 1982.

[3] G. Bessonnet, P. Seguin, and Ph. Sardin. A parametric optimization approach to walking pattern synthesis. International Journal of Robotics Research, 24(7):523-537, 2005.

[4] G. Cabodevila, N. Chaillet, and G. Abba. Energy-minimized gait for a biped robot. In In Proc. AMS, pages 90-99, 1995.

[5] P.H. Channon, S.H. Hopkins, and D.T. Pham. Derivation of optimal walking motions for a bipedal walking robot. robotica, 10(Part 2):165172, 1992.

[6] C. Chevallereau, G. Abba, Y. Aoustin, F. Plestan, E. R. Westervelt, C. Canudas-de Witt, and J. W. Grizzle. Rabbit: A testbed for advanced control theory. IEEE Control System Magazine, 23(5):57-78, 2003.

[7] C. Chevallereau and Y. Aoustin. Optimal reference trajectories for walking and running of a biped. Robotica, 19(5):557-569, 2001.

[8] C. Chevallereau, A. M. Formal'sky, and D. Djoudi. Tracking of a joint path for the walking of an under actuated biped. Robotica, 22(1):15-28, 2004.

[9] C. K. Chow, and D.H. Jacobsoni. Studies of Human Locomotion via Optimal Programming. Mathematical Biosciences, 10:229-306, 1971. 
[10] D. Djoudi, C. Chevallereau, and Y. Aoustin. Optimal reference motions for walking of a biped robot. In Proceedings of IEEE Int Conf. Rob 8 Auto., pages 2014-2019, 2005.

[11] Q Huang, K. Yokoi, S. Kajita, K. Kaneko, Arai H., N. Koyachi, and K. Tanie. Planning walking patterns for a biped robot. IEEE Trans on Robotics and Automation, 17(3):280-289, 2001.

[12] Y. Hurmuzlu. Dynamicla of bipedal gait part; part i: Objective functions and the contact event of a planar of a planar five-link biped. ASME Journal of Applied Mechanics, 60(2):331-336, 1993.

[13] B. Khosravi-Sichani. Control of multi-linkage planar systems in the air and on the control. Phd thesis, The Ohio State University, Colombus, $\mathrm{OH}, 1985$.

[14] P. Kiraikov and W. Schiehlen. CISM Courses, volume 381, chapter On direct-search optimisation of biped walking, pages 134-140. Eds. A. Morecki et al, springer-wien-new-york edition, 1997.

[15] S. Miossec and Y. Aoustin. Dynamical synthesis of a walking cyclic gait for a biped with point feet. Fast motions in biomlechanics and robotics, Lecture Notes in Control and Information Sciences, Springer-Verlag, 2006.

[16] C. F Rose, Cohen M. F., and B. Bodenheimer. Verbs and adverbs: Multidimensional motion interpolation. IEEE Computer Graphics and Applications, 114:32-40, 1998.

[17] M. Rostami and G. Bessonnet. Impactless sagittal gait of a biped robot during the single support phase. In Proceedings of IEEE Int Conf. Rob $E$ E Auto., pages 1385-1391, 1998.

[18] L. Roussel, C.. Canudas-de Witt, and A. Goswani. Generation of energy optimal complete gait cycles for biped. In Proceedings of IEEE Int Conf. Rob \& Auto., pages 2036-2042, 1998.

[19] M.W. Spong and M. Vidyasagar. Robot dynamics and control. John Wiley, New-York, 1991.

[20] D. H. Sutherland, K. R. Kaufman, and J. R. Moitoza. Kinematics of normal human gait. Human Walking, Eds. Jessica Rose and James G. Gamble, Williams and Wilkings, 349p, 1994.

[21] C. L. Vaugham, B. L. Davis, and J. C. O'Connor. Dynamic of humain gait. Kiboho Publishers, Cape Town, South Africa, 1999.

[22] K. R. Vaughan. Biomechanics of running gait. Crit. Rev. Eng., 12(1):148, 1984.

[23] E.R. Westervelt, J.W. Grizzle, C. Chevallereau, J-h. Choi, and B. Morris. Feedback Control of Dynamic Bipedal Robot Locomotion. Taylor \& Francis/CRC Press, 2007.

[24] F. Zonfrilli, M. Oriolo, and T. Nardi. A biped locomotion strategy for the quadruped robot sony ers-210. In Proc. IEEE Int. Conf. on Robotics and Automation ICRA, pages 2768-2774, Washington, D.C., USA, 2002. 